\title{
Habituation and rehabituation of the electrodermal orienting reflex in Macaca fascicularis monkeys as a function of stimulus variability
}

\author{
H. D. KIMMEL and M. R. MURRIN \\ University of South Florida, Tampa, Florida 33620
}

\begin{abstract}
Two groups of six adult female Macaca fascicularis monkeys received repeated presentations of either a fixed pure tone or a tone that varied in frequency from trial to trial, until their skin conductance response habituated. The monkeys were then rehabituated with a new, unchanging stimulus. The monkeys showed a small but insignificant tendency towards retention of habituation, but no savings in either SCR magnitude or latency scores. The habituation-retardation effect, seen in humans' trials-to-criterion data and to some extent in SCR magnitude and latency measures in Cebus monkeys, was suggested in the macaques' trials data but not in their magnitude or latency data. A significant accelerative change in heart rate was elicited by the tones, unlike the decelerative response seen in humans, Macaca arctoides, and Cebus monkeys. The Macaca fascicularis monkeys showed a change toward HR deceleration with repeated, unchanging stimulation, but not with varying stimulation. Also, a shift in auditory frequency following habituation resulted in a HR increase following initial habituation to a fixed stimulus, but not following habituation to variable stimuli. It could not be determined whether the difference in HR response between the present macaques and previously studied Cebus monkeys was due to species or sex, since the macaques in this study were all female, while the Cebus monkeys in our earlier study were all male. Both species appear to provide some support for the matching model of habituation.
\end{abstract}

The electrodermal orienting reflex (OR) in humans habituates under repeated auditory stimulation in fewer trials when the repeated tone is unchanging in all respects than when it changes in frequency from trial to trial (Kimmel, Piroch, \& Ray, 1979). This habituationretardation effect of variability in tone frequency was not found in a recent study with adult male Cebus albifrons monkeys (Kimmel, Raich, \& Brennan, 1979). The Cebus monkeys were divided into two groups, one that received a fixed pure tone on every trial and another that received a tone that varied in frequency from trial to trial. Following habituation of the skin conductance response (SCR) to a criterion of two successive zero responses, both groups of monkeys were shifted to a new, unchanging tone for rehabituation. While there was a significant reduction from habituation to rehabituation in the number of trials to criterion, in SCR magnitude, and in the reciprocal of SCR latency (i.e., demonstrating "retention" of habituation), there were no differences in these measures between the fixed and variable groups. The monkeys' sensitivity to changes in tone frequency was manifested in the observation that the average

This work was done under a grant from the U.S. Army Medical R and D Command, No. 17-C-76-6053. R. Paul Taylor provided technical assistance.
SCR magnitude declined from the first to the second trial of habituation and rehabituation in the fixed group but not in the variable group.

The present study was a replication of the Kimmel, Raich, and Brennan (1979) procedures with a different species of monkey, Macaca fascicularis. Since the Old World macaque monkeys are more phylogenetically advanced than the New World Cebus animals, it was thought that they would be more likely to show the SCR habituation-retardation effect commonly seen in humans.

\section{METHOD}

\section{Subjects}

Twelve female adult Macaca fascicularis monkeys served as subjects. These animals were naive to habituation or conditioning experiments and had not previously experienced pure tones. They were divided randomly into two groups of six.

\footnotetext{
Apparatus

During data collection, the monkey was restrained in a custommade Plexiglas primate chair, previously described in detail by Kimmel, Raich, and Brennan (1979).

The SCR was picked up from the plantar surfaces of the monkeys' feet by zinc-zinc sulfate electrodes in Teflon cups filled with saline electrode jelly (recipe given in Grings, 1954). The monkey's two plantar surfaces served as bipolar resistors in a constantvoltage bridge circuit (Venables \& Christie, 1973), whose output voltage was proportional to the animal's skin conductance. This
} 
signal was digitized for input into a Data General Nova 2 computer.

Electrocardiogram (ECG) signals were obtained via two K \& G pediatric foam stress-test electrodes attached to the chest in shaved locations predetermined to yield good signal strength. The ECG was amplified and filtered so that the peak of the $\mathbf{R}$-wave initiated a digital input to the computer.

The computer was programmed to administer the stimuli and to search for SCRs during the period beginning $1.0 \mathrm{sec}$ after stimulus onset and ending $5.0 \mathrm{sec}$ later. The computer also scored SCR magnitude and latency for the first response detected during this interval. For the digitized ECG signal, the computer timed interbeat intervals, generating a baseline heart-rate (HR) measure averaged for the 10 -sec period prior to the administration of each stimulus and printing out a series of interbeat intervals for at least the succeeding 6 sec. A 10-sec baseline HR was used to ensure its stability. Since the Macaca fascicularis' average HR generates approximately 4 beats/sec, the elicited HR response is based upon some 24 poststimulus heartbeats, compared with 22 used in Kimmel, Raich, and Brennan's (1979) study with Cebus monkeys.

The chair containing the monkey was placed inside a dimly lighted, sound-attenuated chamber $(86.7 \mathrm{~cm}$ wide $\times 73.7 \mathrm{~cm}$ deep $\times 86.7 \mathrm{~cm}$ high). A speaker behind the animal's head delivered continuous white noise at $70 \mathrm{~dB}$ (physical reference) for masking purposes. The white noise and the pure tone stimuli were produced by a Grayson-Stadler audio generator and were presented via the same speaker. Tone intensity was $80 \mathrm{~dB}$ (physical reference). Thus, the administration of the auditory stimuli was identical to the Kimmel, Raich, and Brennan (1979) study. Tone frequencies ranging from 800 to $1,600 \mathrm{~Hz}$ were used, depending on the experimental condition; tone duration was $5.0 \mathrm{sec}$. The stimuli were delivered at time intervals ranging in $5.0-\mathrm{sec}$ steps from 20 to $60 \mathrm{sec}$, with a mean of $40 \mathrm{sec}$, in a randomized sequence. The shortest and longest intervals occurred on only about $10 \%$ of the trials, on the average.

\section{Procedure}

The monkeys were habituated to handling for some 2 months prior to the beginning of a 2-week period of habituation to sitting in the primate chair and response measurement. Half of the monkeys were assigned at random to the fixed group, which received a $1,200-\mathrm{Hz}$ pure tone until two successive presentations occurred without a detectable SCR. Half of these monkeys were then shifted to a $1,000-\mathrm{Hz}$ pure tone, while the others were shifted to a $1,400-\mathrm{Hz}$ tone for rehabituation. The remaining six monkeys, the variable group, were initially presented with 1,000-, 1,200-, and $1,400-\mathrm{Hz}$ tones in a random sequence, except that each frequency occurred once in the first three trials and none was presented more than twice in succession. The habituation criterion for this group was also two successive nonresponses. For rehabituation, half of the animals in this group were shifted to a $1,600-\mathrm{Hz}$ tone, while the remaining half were shifted to an $800-\mathrm{Hz}$ tone. The rehabituation criterion for all groups was, again, two successive nonresponses.

\section{RESULTS}

The average numbers of trials required to reach the habituation and rehabituation criteria in the fixed and variable groups are shown in Table 1 . The apparent retardation of habituation due to stimulus variability was not significant. The tendency for trials to criterion to decrease from habituation (mean $=$ 11.58 ) to rehabituation (mean $=6.42$ ) also failed to attain significance $[F(1,10)=3.45, p<.10]$.

Since habituation was fairly rapid, only the first two trials of habituation and rehabituation were used to evaluate the magnitude and latency of the SCR
Table 1

Average Numbers of Trials to Criterion in Habituation and Rehabituation Under Fixed and Variable Stimulus Conditions

\begin{tabular}{lcc}
\hline & Habituation & Rehabituation \\
\hline Fixed & 8.83 & 6.50 \\
Variable & 14.33 & 6.33 \\
\hline
\end{tabular}

Table 2

Average SCR Magnitude (in Micromhos) and Reciprocal of Latency (1/Sec) on Trials 1 and 2 of Habituation and Rehabituation for the Fixed and Variable Groups

\begin{tabular}{lrccr}
\hline & \multicolumn{2}{c}{ Habituation } & & \multicolumn{2}{c}{ Rehabituation } \\
\cline { 2 - 4 } & Trial 1 & Trial 2 & Trial 1 & Trial 2 \\
\hline & \multicolumn{4}{c}{ Magnitude } \\
Fixed & .86 & 1.04 & 1.10 & .52 \\
Variable & 1.32 & 1.19 & 1.08 & 1.36 \\
& \multicolumn{4}{c}{ Reciprocal of Latency } \\
Fixed & .54 & .45 & .50 & .52 \\
Variable & .65 & .61 & .62 & .64 \\
\hline
\end{tabular}

elicited by the tone. Table 2 shows the average SCR magnitude (micromhos) and the average reciprocal of latency $(1 / \mathrm{sec})$ on the first two trials of habituation and rehabituation in both groups. Neither SCR magnitude nor latency showed any significant effects, contrary to our earlier results obtained with Cebus albifrons monkeys and with humans.

Cardiac interbeat intervals obtained during six consecutive poststimulus seconds were converted offline to heart rate (HR) in beats/min for each of the first six 1-sec periods following stimulus onset (approximately 4 beats/sec). These measures were evaluated in relation to the average baseline HR measures obtained during the 10 -sec prestimulus period. This analysis showed that there was a significant increase in HR over the 6-sec period for all conditions $[\mathrm{F}(5,60)=4.11, \mathrm{p}<.01]$, with a peak at the 4 -sec period. The second-by-second course of this average change in HR is presented in Table 3.

Trend analysis of the overall course of elicited HR change showed both the upward linear component $[F(1,60)=15.38, p<.01]$ and the inverse U-shaped quadratic component $[\mathrm{F}(1,60)=7.85, \mathrm{p}<.01]$ to be significant. A more detailed presentation of the average HR changes from the prestimulus to the poststimulus periods is given in Table 4. These HR measures were obtained during the 10-sec prestimulus and 6-sec poststimulus periods.

The slight difference between the fixed and variable groups' HRs was not statistically significant. There was some tendency for HR to decline between habituation and rehabituation $[F(1,10)=3.42, p<.10]$. The three-way interaction between pre- and poststimulus $\mathrm{HR}$, habituation and rehabituation, and first and second trials was significant $[F(1,10)=9.47, p<.05]$, 
Table 3

Average Chance in Heart Rate in Macaca fascicularis Following Onset of Auditory Stimulus (in Beats per Minute)

\begin{tabular}{ccccccc}
\hline & \multicolumn{6}{c}{ Seconds Following Stimulus Onset } \\
\cline { 2 - 7 } & 1 & 2 & 3 & 4 & 5 & 6 \\
\hline Heart Rate Change & .49 & 2.00 & 2.09 & 2.42 & 2.26 & 1.92 \\
\hline
\end{tabular}

Table 4

Average Heart Rate Before and After (Pre vs. Post) Administration of Auditory Stimulus on Trials 1 and 2 of Habituation and Rehabituation in Fixed and Variable Groups

\begin{tabular}{|c|c|c|c|c|}
\hline \multirow[b]{2}{*}{ Trial } & \multicolumn{2}{|c|}{ Habituation } & \multicolumn{2}{|c|}{ Rehabituation } \\
\hline & Pre & Post & Pre & Post \\
\hline & \multicolumn{4}{|c|}{ Fixed Group } \\
\hline \multirow[t]{2}{*}{$\begin{array}{l}1 \\
2\end{array}$} & $\begin{array}{l}218 \\
214\end{array}$ & $\begin{array}{l}222 \\
211\end{array}$ & $\begin{array}{l}214 \\
209\end{array}$ & $\begin{array}{l}211 \\
215\end{array}$ \\
\hline & \multicolumn{4}{|c|}{ Variable Group } \\
\hline $\begin{array}{l}1 \\
2\end{array}$ & $\begin{array}{l}238 \\
235\end{array}$ & $\begin{array}{l}239 \\
238\end{array}$ & $\begin{array}{l}229 \\
230\end{array}$ & $\begin{array}{l}230 \\
231\end{array}$ \\
\hline
\end{tabular}

as was the more complex interaction of this effect with groups $[\mathrm{F}(1,10)=17.78, \mathrm{p}<.01]$.

This complex interaction appears to reflect the fact that the fixed group's accelerative HR response changed to deceleration on the second presentation of the fixed stimulus, while the variable group's somewhat smaller accelerative response became more accelerative on the second trial, with a changed stimulus-all of this during habituation. After receiving one presentation of a changed stimulus in rehabilitation, the fixed group's decelerative response on the first trial became accelerative on the second trial. The variable group's HR changed very little during the first two trials of rehabituation. Of course, these significant interactions do not establish that any of the simpler contrasts were separately significant.

\section{DISCUSSION}

The present Macaca fascicularis monkeys took fewer trials to reach the criterion of two successive nonresponses during rehabituation than during initial habituation, but this effect failed to attain significance. This effect was significant in a previous study using Cebus albifrons monkeys (Kimmel, Raich, \& Brennan, 1979). Retention of habituation is commonly seen in humans. Although the data presented in Table 1 suggest a possible habituation-retardation effect due to stimulus variability, this effect was also not significant. A similar nonsignificant tendency was observed in our Cebus monkeys.

Unlike the Cebus monkeys, the present macaques also did not show the stimulus variability effect in either their SCR magnitude or latency data. Thus, while both species showed the stimulus variability effect to some degree, the macaques demonstrated it only marginally in their average number of trials to criterion, while the Cebus monkeys showed the effect significantly in their SCR magnitude and latency data. Thus, neither species exhibited the habituationretardation effect unambiguously or convincingly.

An unexpected difference between the present results and those of our earlier study with Cebus monkeys was in the patterns of change in HR elicited by the stimuli. The HR change during the first $6 \mathrm{sec}$ following the stimulus was decelerative in Cebus albifrons but accelerative in the present data of Macaca fascicularis, despite the fact that identical stimuli were used in the two studies. The slight habituation observed in the Cebus monkeys' HR responses was in the direction of less deceleration. Habituation of elicited HR changes in Macaca fascicularis, on the other hand, tended to be in the direction of less acceleration. Table 4 shows that repetition of the stimulus from Trial 1 to Trial 2 of habituation in the fixed group resulted in a shift from acceleration to deceleration. In the variable group, there was a slight acceleration on Trial 1 and, with a changed stimulus, greater acceleration on Trial 2. Both groups had significantly reduced rates in rehabituation as compared with habituation (about $10 \mathrm{bpm}$ slower). Changing the frequency of the fixed group's stimulus between habituation and rehabituation was followed by a return to acceleration on the second trial. These findings suggest that Macaca fascicularis' cardiac OR may be accelerative rather than decelerative, as is true for humans (Graham, 1979), Cebus albifrons (Kimmel, Raich, \& Brennan, 1979), and, to some extent, for Macaca arctoides (Weisbard \& Graham, 1971). Since both of our studies used identical stimulus intensities (as well as other parameters), it does not seem likely that defensive reactions were being made in the present study.

It is possible that the differences we have found between adult male Cebus albifrons and adult female Macaca fascicularis animals are due not to species but to sex. We are presently unable to disentangle the species and sex factors, since they were completely confounded between our two studies. In our human electrodermal OR habituation studies, we have found that males make larger SCRs than do females and require greater numbers of trials to habituate (Kimmel \& Kimmel, 1965). Male humans also are more likely to show the SCR habituation-retardation effect of stimulus variability than are females (Castleman, Brennan, \& Kimmel, 1979). The present macaques were all female but required more trials to attain habituation of the SCR than did the male Cebus monkeys; in addition, they showed no reduction in SCR magnitude across trials or between habituation and 
rehabituation (while male Cebus monkeys did), they did not show longer latencies of SCR in rehabituation as compared with habituation (as Cebus did), and they made an elicited HR response that was accelerative up to $4 \mathrm{sec}$ (while Cebus' pattern was decelerative). Male and female Macaca arctoides tend to show a decelerative component in their elicited HR changes (Weisbard \& Graham, 1971).

Unsystematic observations made while handling animals in our laboratory suggest that the male Cebus monkeys are far more aggressive than are the female Macaca fascicularis. The male Cebus violently resists transfer from the home cage to the primate chair and requires two experimenters for this task. The female Macaca fascicularis is far easier to move and is almost never violent, although possibly just as traumatized. For reasons of this type, it would be premature to attribute the present differences either to sex or to species alone. In any case, the female macaques' HR reaction was primarily accelerative in this study, whether due to sex, species, or whatever. Whether these reactions were ORs or DRs cannot be definitively stated, although the overall pattern of the present findings points in the direction of ORs.

Aside from the difference in HR responses in the two species we have studied, our combined results are more or less in accord with a matching theory of OR habituation. The failure to obtain significant results in all expected places may be attributed in part to small sample sizes (seven vs. seven Cebus and six vs. six macaques). Stimulus factors and the effect of handling and immobility in the primate chair must also be considered, even though the animals in both of our studies received substantial habituation to handling and placement in the apparatus. In spite of these complicating factors, the results of these experiments show that substantial commonality among primate species exists in OR habituation.

\section{REFERENCES}

Castleman, K., Brennan, A. F., \& Kimmel, H. D. The effect of stimulus variability on $O R$ habituation in male and female humans. In H. D. Kimmel, E. H. van Olst, \& F. J. Orlebeke (Eds.), The orienting reflex in humans. Hillsdale, N.J: Erlbaum, 1979.

Graham, F. K. Distinguishing among orienting, defense, and startle reflexes. In H. D. Kimmel, E. H. van Olst, \& J. F. Orlebeke (Eds.), The orienting reflex in humans. Hillsdale, N.J: Erlbaum, 1979.

Grings, W. W. Laboratory instrumentation in psychology. Palo Alto, Calif: National Book Company, 1954.

Kimmel, H. D., \& Kimmel, E. Sex differences in adaptation of the GSR under repeated applications of a visual stimulus. Journal of Experimental Psychology, 1965, 70, 536-537.

Kimmel, H. D., Piroch, J., \& RAY, R. L. Monotony and uncertainty in the habituation of the orienting reflex. In H. D. Kimmel, E. H. van Olst, \& J. F. Orlebeke (Eds.), The orienting reflex in humans. Hillsdale, N.J: Erlbaum, 1979.

Kimmel, H. D., Raich, M. S., \& Brennan, A. F. Habituation and rehabituation of the monkey's orienting reflexes as a function of stimulus variability. Physiological Psychology, 1979, 7, 283-286.

Venables, P. H., \& Christie, M. J. Mechanisms, instrumentation, recording techniques and quantification of response. In W. F. Prokasy \& D. G. Raskin (Eds.), Electrodermal activity in psychological research. New York: Academic Press, 1973.

Weisbard, C., \& Graham, F. K. Heart-rate change as a component of the orienting response in monkeys. Journal of Comparative and Physiological Psychology, 1971, 76, 74-83.

(Received for publication July 11, 1980; revision accepted February 17, 1981.) 